\title{
Comparison of administration of platelet concentratessuspendedin M-sol or BRS-A for pediatric patients
}

\author{
Shunsuke Kojima ${ }^{1}$, Ryu Yanagisawa ${ }^{1,2}$, Miyuki Tanaka ${ }^{3}$, Yozo Nakazawa $^{3}$, and Shigetaka \\ Shimodaira ${ }^{4}$ \\ ${ }^{1}$ Division of Blood Transfusion, Shinshu University Hospital, Matsumoto, Japan \\ ${ }^{2}$ Center for Advanced Cell Therapy, Shinshu University Hospital, Matsumoto, Japan \\ ${ }^{3}$ Department of Pediatrics, Shinshu University School of Medicine, Matsumoto, Japan \\ ${ }^{4}$ Department of Regenerative Medicine, Kanazawa Medical University, Uchinada-Cho, Kahoku-Gun, \\ Japan
}

\section{CONFLICT OF INTEREST}

The authors have no potential conflicts of interest to declare.

Corresponding author: Ryu Yanagisawa, MD, PhD

Division of Blood Transfusion, Shinshu University Hospital

3-1-1, Asahi, Matsumoto 390-8621, Japan

Tel: +81-263-373240; Fax: +81-263-373027

E-mail: ryu@shinshu-u.ac.jp

Word count: 2550

\section{RUNNING TITLE}

Resuspended PC in M-sol or BRS-A 


\begin{abstract}
BACKGROUND: Two different platelet additive solutions (PASs), such as M-sol and BRS-A, used in succession have been reported as novel PASs in Japan. However, there are not enough clinical data comparing platelet concentrates (PCs) suspended in these PASs.
\end{abstract}

STUDY DESIGN AND METHODS: A retrospective cohort study of consecutive cases was performed between 2013 and 2018. For the first 30 months, children with primary hematologic and/or malignant diseases were transfused resuspended PC in Msol (RPC-M) as plasma-replaced PCs. For the latter 30 months, children were transfused plasma-replaced PC in BRS-A (RPC-B) under the same conditions. Children transfused with conventional PCs (containing residual plasma) were defined as controls. We evaluated the frequency of adverse events, corrected count increment (CCI), and bleeding occurrence in the children.

RESULTS: Overall, 84 patients received 679 conventional PC transfusions. Allergic transfusion reactions (ATRs) occurred in $12(14.3 \%)$ patients transfused with $12(1.8 \%)$ bags. Fifty-nine patients received 1182 bags of RPC-M, andonepatient(1.7\%)hadfive $(0.4 \%)$ episodes of ATRs. During the latter study period, 58 patients were transfused 1044 bags of RPC-B, with ATRs occurring in four (6.9\%) patients transfused with four (0.4\%) bags. No other adverse events were observed with either RPC-MorRPC-B.

CCIs (24 h) were not significantly different for the three different PCs, and posttransfusion bleeding was not observed.

CONCLUSION: RPC using two different PAS in children appeared to prevent ATRs accompanied without othernon-allergicevents. Because transfusion efficacy was not significant, either of the PASs could be used with equivalent results based on the 
clinical situation.

KEY WORDS: allergic transfusion reaction, bicarbonated Ringer's solution, platelet additive solutions, resuspended platelet concentrate 


\section{INTRODUCTION}

The effectiveness of washed platelet concentrate (PC) in reducing adverse reactions associated with PC transfusion, including allergic transfusion reactions (ATRs), has been clarified in previous reports. ${ }^{1-5}$ Good platelet additive solutions (PASs) are essential for washing platelets. ${ }^{2,6,7}$ Although successful use of PASs has been reported in several countries, ${ }^{6,7}$ PAS is still not commercially available in Japan. It was recently reported that M-sol and BRS-A in succession can be used as PAS. ${ }^{8-10}$ In Japan, these solutions are prepared by mixing commercially available products, ${ }^{8-10}$ and their clinical application is currently being tested. ${ }^{1,3,5} \mathrm{M}$-sol requires a mixture of five kinds of commercially available solutions and can be preserved for a year using a special method. ${ }^{3,8}$ Conversely, BRS-A has the advantage of being easily adjusted by mixing two bicarbonated Ringer's solutions with ACD-A solution. ${ }^{9,10}$ Acomparisonofwashed PC using M-sol and BRS-A invitro has confirmed that maintenance effect of platelet quality can be achieved at the same level. ${ }^{11}$ Washed PCs using the classical PAS (G-sol) have been used for many years in Japan, ${ }^{2,3,5}$ and the manual washing method was considered acomplicated method. ${ }^{2,3,5}$ Further, resuspended PC in M-sol or BRS-A as plasma-replaced PC (RPC-M or RPC-B) adjusted by a simplified manual method was verified for use in pediatric patients to prevent ATRs in PC transfusion. ${ }^{3,5}$ According to these results, we confirmed that RPC-M or RPC-B was just as effective as washed PC with G-sol in preventing ATRs, and the transfusion effect (evaluated with corrected count increment, $\mathrm{CCI}$ ) had similar effectiveness as regular PC in plasma. However, because CCI with washed PC with G-sol was inferior to RPC-M or RPC-B, the use of RPC-M or RPC-B was considered preferable. ${ }^{3,5}$ On the other hand, the superiority of RPC-M or RPC-B over the other is still unknown. Currently, although M-sol or an 
equivalent PAS such as BRS-A is recommended in the guidelines of the Japan Society of Transfusion Medicine and Cell Therapy for preparing washed PC, ${ }^{5}$ it is unclear which PAS should be considered as priority. Therefore, it is necessary to verify washed PC comparing two different PASs.

We prepared washedPCsandRPCs for several years at Shinshu University Hospital. Through the years, the prepared PC products have changed from washed PC with G-sol to RPC-M and to the present RPC-B. Therefore, we performed a retrospective cohort study to compare the efficacy of ATR prevention, frequency of non-allergicevents, and transfusion effect in RPCs prepared using the same method with two different PAS.

\section{MATERIALS AND METHODS}

\section{Patient selection}

A retrospective cohort study was performed between April 2013 and March 2018, during which time patient data were collected consecutively as representative samples. Eligible patients met the following inclusion criteria: aged $\leq 20$ years; diagnosis of primary hematologic and/or malignant disease; or thrombocytopenia due to primary disease, chemotherapy, or hematopoietic stem cell transplantation. In this study, RPC was provided regardless of the patient's history of ATRs. Based on the method of indication guidance for washed and replaced platelets and their preparation, we prepared a simplified RPC-M (i.e., resuspended PC in M-sol, which differs from the classical

method with wash and replaced PC) $)^{3,5}$ as a plasma-replaced PC between April 2013 and September 2015. During the latter part of the study, we prepared RPC-B using the same methods. Therefore,there were no other differences in the comparison between the two types of RPCs except in terms of suspended PASs and prepared periods. Because RPC 
could not be prepared during off-shifts (i.e., night shifts, weekends, and holidays), 84 patients received transfusions of 679 untreated regular PC in plasma (P-PC transfusion) over the total study period. We defined these P-PC transfusions as the control. Patient characteristics in each group are shown as Table 1. The Institutional Review Board of Shinshu University School of Medicine approved this cohort study, and written informed consent was signed by the patients' parent or guardian before the transfusion.

\section{Preparation and transfusion of P-PCs, RPC-M, and RPC-B supporting medications for transfusion}

All transfused regular PC products were obtained from single-donor apheresis from random donors based on blood type. ${ }^{12}$ The first aliquot of blood was discarded to prevent bacterial contamination and skin fragment contamination from the needle puncture site. ${ }^{13-15}$ As previously described, pre-storage leukocyte reduction was also performed to reduce adverse transfusion effects. ${ }^{5,16}$ Six type of PC products exist based on the number of platelet contained:1,2,5,10,15,and 20 units. ${ }^{15}$ Allpatientsinthis study were transfused with 10 units of regular PCs in plasma, including $2 \times 10^{11} / \mathrm{L}$ platelet regardless of body weight, which is the standard unit is the most frequently used in Japan;conversely,1-2 units are used for infants or neonates. ${ }^{15}$ All regular PCs in plasma were supplied following nucleic acid testing for HBV, HCV, and HIV at the Japanese Red Cross Blood Center; thereafter, the components were exposed to irradiation ranging from 15-50 Gy and used within 4 days of apheresis. ${ }^{5,16}$ RPCs were prepared at the Shinshu University Hospital according to Japan Society of Transfusion Medicine and Cell Therapy guidelines. ${ }^{5}$ BRS-A and M-sol as PASs were prepared based on a method described previously. ${ }^{5,9}$ Premedication was not routinely 
administered before transfusion of platelets in PAS M-sol and BRS-A. Intravenous medicine with antihistamine was administered 5 min prior to transfusion only for P-PC transfusions.

\section{Evaluation of ATRs and transfusion efficacy}

Reactions to the transfused platelets were recorded during the transfusion and follow-up periods, including data regarding the type of adverse reaction (ATR and non-allergic event), bleeding status on the date of PC transfusion, transfusion-associated infections, sepsis due to bacterial contamination in blood components, and transfusion-related viral infections such as $\mathrm{HBV}, \mathrm{HCV}$, syphilis, and $\mathrm{HIV}^{3}$ All reactions recognized by physicians and/or nurses were reported to the Division of Blood Transfusion at our institution. The types of ATRs were assessed as described previously. ${ }^{3}$ ATR was defined as the presence of at least one of the following during or within $4 \mathrm{~h}$ of transfusion: maculopapular rash with/without pruritus; urticaria; pruritus; generalized flushing; localized angioedema; edema of the lips, tongue, and uvula; erythema and edema of the periorbital area; conjunctival edema; respiratory distress; bronchospasm; and hypotension. We calculated the post-transfusion CCI $(24 \mathrm{~h})\left(\times 10^{10} / \mathrm{L}\right)$ to evaluate the transfusion effect based on a previously described method. ${ }^{5}$ CCI was evaluated in each patient within 6 months of the initial PC transfusion for a maximum of $10 \mathrm{PC}$ products. We excluded transfusions from analysis in which aggravation of the primary disease resulted in refractoriness to transfusion, fever, infection, under the immunological responses such as graft-versus-host disease, disseminated intravascular coagulation, splenomegaly, or bleeding/bleeding tendency. 


\section{Quality evaluation of RPC-M, and RPC-B}

We analyzed the composition and platelet activation tests of RPCs manufactured in our institution using two different PAS (M-sol and BRS-A). We calculated platelet recovery and plasma protein reduction based on platelet counts and total protein contents.

Furthermore, $\mathrm{pH}$ and concentrations of total protein, albumin, sodium, potassium, chlorine, calcium, phosphorus, magnesium, and glucose were analyzed. We also evaluated P-selectin (CD62P) positivity as a platelet activation marker.

\section{Statistical analysis}

Fisher's exact test was used to determine the difference in characteristics of patients transfused with each PC product and assess the differences in ATR ratio for the different PC products. ATRs were evaluated by the frequency of each incidence per patients and bags between P-PC and RPC with BRS-A or M-sol. We used the KruskalWallis test to evaluate the effect of transfusion response in terms of 24-h CCI for each PC and the difference in patient ages between each group. Unpaired t-test was used to analyze the difference with the quality inspection of RPC with two different PASs. All

statistical analyses used EZR software. ${ }^{17}$ Statistical significance was defined as $\mathrm{P}<0.05$.

\section{RESULTS}

\section{ATR frequency in P-PC, RPC-M, and RPC-B}

During the study period, ATR soccurred in $12(14.3 \%)$ of 84 patients from $12(1.8 \%)$ of 679 bags of P-PCs, in 1 (1.7\%) of 59 patients from 5 (0.4\%) of 1182 bags of RPC-M, and in $4(6.9 \%)$ of 58 patients from $4(0.4 \%)$ of 1044 bags of RPC-B. There were no differences in terms of patient background among the groups (Table1). Moreover, ATR 
frequency per patient and per product was different in the three PC products (Table 2). ATRs from RPC-MorRPC-B were not frequent compared with those in control P-PCs for patients and products. However, there was no significant difference between RPC-M and RPC-B per patient and product.

\section{Transfusion effectiveness among the PC products}

We saw no pre- or post-transfusion hemorrhage with any of the transfused platelets, including control P-PC and RPC-M or RPC-B. CCIs $\left(\times 10^{10} / \mathrm{L}\right)$ at $24 \mathrm{~h}($ median $\pm \mathrm{SD})$ are shown in Table 2. The CCI of the control P-PC was higher than either RPC-Mor RPC-B. However, the three products were not significantly different.

\section{Frequency of non-allergicevents associated with RPC}

There were non-allergic events associated with transfusion or products of RPC.

\section{The quality inspection of the RPCs using M-soland BRS-A}

Table 3 shows the quality assessment of RPCs using M-sol and BRS-A prepared at our institution. PCs before the plasma-replacing process with each PASs are shown as P-PCs. Each score is shown as mean and standard deviation. Although the compositions differed among RPC products according to each PAS, the levels of platelet recovery, plasma protein reduction, and P-selectin were similar.

\section{DISCUSSION}

Although the remarkable difference between the control group and the two groups receiving RPC-M and RPC-B was that the control patients were premedicated, we 
confirmed that RPC-M or RPC-B had similar ATR prevention effects and comparable transfusion effects when compared with control P-PCs without the occurrence of nonallergicevents. To effectively prevent ATRs with PC transfusion, it is important to sufficiently remove the plasma components during preparation of the washed PC. ${ }^{2}$ In our manual method of RPC adjustment, it is possible to remove approximately $90 \%$ $95 \%$ of plasma proteins in the preparation process, ${ }^{3,5}$ but it is difficult to remove as many plasma proteins (approximately 99\%) as obtained from reported automated equipment methods. ${ }^{11}$ However, together with evidence from previous reports targeting the same disease population of pediatric patients, ${ }^{3,5}$ our manual method of RPC preparation can prevent ATRs effectively regardless of the type of PAS (M-sol or BRS-A). Although ATRs continued to occur, even after introducing RPC transfusions, the range of ATRs was small. In our institution, after introducing the RPC, no transfusion with RPC has been forced to stop due to a severe ATR although some cases develop similar minor allergic symptoms. In addition, because no other adverse events were associated with preparing RPC using M-sol or BRS-A, it was considered that both of RPCs with two different PAS have comparable safety profiles.

Conversely, there are concerns about poor transfusion effects in performing washed PC transfusion; ${ }^{18}$ this is presumed to involve the loss of platelets accompanying the washing operation and shortening of life span due to activation of platelet activation. ${ }^{3}$ Although the RPCs in our study were prepared with two different PAS using the same method, there were no significant differences in CCI scores. Therefore, it appears that there is no influence of CCI decrease regardless of which PAS is used. It is necessary to include magnesium, bicarbonate, potassium, calcium, glucose etc., in PAS in order to maintain the quality of washed PC. ${ }^{6,11,19-21}$ Although the composition contents are not 
the same for the two types of PAS, all of these conditions required for PAS are satisfied. ${ }^{11}$ In vitro verification of the quality maintenance effect of platelets is reported to be almost equal for either type of PAS. ${ }^{11}$ Therefore, we are satisfied that there is no significant difference in transfusion effect between RPC-M and RPC-B even in actual clinical practice. Although clinical influences associated with the selection of P-PC and RPC with M-sol or BRS-A did not seem to differ except for the frequency of ATRs, actual quality inspection of each PC products were different as shown in Table 3. These inspections may differ according to the content of each PASs, such as the amount of anticoagulant agent, residual plasma levels, and original platelet quality. Furthermore, differences between institutions or instruments may also have an effect. Although it may be difficult to establish a standard of quality inspection for each PC product, selection of an appropriate PAS as per the pediatric patient' status or disease should be considered in future.

Preparing washed PC can prevent the occurrence ATRs of transfusion by eliminating factors contained in the plasma, but another patient's own characteristics which associated to develop ATRs may present. ${ }^{16,22}$ As this study was conducted only in children, it is reported that transfusion adverse reactions are likely to occur more frequently in children than in adults, ${ }^{23,24}$ and particular attention should be paid to the high occurrence of ATR and febrile non-hemolytic transfusion reactions accompanying PC transfusion. ${ }^{16,23,24}$ Not only washed PCs, ${ }^{1,25}$ but attempts of preventing adverse transfusion reactions by introducing concentrated or plasma reduced PC (CPC) and plasma-replaced PC stored with PAS at residual plasma in 30\%-35\% (PC in PAS) were also reported, primarily in the adult population. ${ }^{26-29}$ We have reviewed the recent reports stating the use of washed PCs, RPCs, CPCs, and PCs in PAS in comparatively large 
number of children or neonates (Table 4). ${ }^{3,5,26,30,31}$ Several previous studies have analyzed these data in both pediatric and adult populations, ${ }^{4,18,32,33}$ however, thus far, only few studies have targeted only the pediatric population. Furthermore most of the studies in children were planned for retrospective analysis. Although at present there is very little evidence in children, no serious side effects have been reported. It is believed that reduction in side effects can also be expected even in children by using such PC formulations, including our RPC method. ${ }^{3,5,26}$ Although introducing washed PC, RPC, $\mathrm{CPC}$, or PC in PAS may be useful even in children, it is essential to clarify the Effectiveness of each PC formulation to prevent ATRs inprospective studies involving large populations and to determine which transfusion method is suitable for each population and disease status. Incontrast, similar to our study, most of theprevious reports were planned with the purpose of prophylactic transfusions. It is necessary to (1) evaluate using multiple evaluation items, including hemostatic effect, in the future and (2)verify whether there is a difference in transfusion effect among the different PC products. Another limitation of our study is that our data were not independent as some of the patients included in the control group were also transfused in the M-sol and BRS-A groups. Further, aformal samplesize calculation was not performed, suggesting that the study was underpowered to detect asignificant difference between the treatment groups.

RPC using two different PASs in children appears to prevent ATRs accompanied Without other adverse events. Because transfusion efficacy was not significant, either of the PASs can be used with equivalent results. Compared with conventional PAS, M-sol is reported to be superior in several reports, ${ }^{2,5,8}$ but a learning curve is involved.

Compared with M-sol, BRS-A is easy to adjust and non-inferiority was also confirmed 
in RPC-B transfusion. In addition, because automated adjustment has already been introduced in clinical use, ${ }^{34}$ future developments may be expected. Although the efficacy and safety of RPC-B transfusion, including that reported this study, are gradually being reported, sufficient evidence has yet to be confirmed. It appears that prospective studies targeting the majority of cases are necessary to expand adaptive diseases and verify adult cases in the future. 
Table 1. Differences in characteristics of patients transfused with each PC product

\begin{tabular}{|c|c|c|c|c|}
\hline \multirow[b]{2}{*}{ Patient characteristic } & \multicolumn{3}{|c|}{ Transfused PC products } & \multirow[b]{2}{*}{ p-value } \\
\hline & P-PC & RPC-M & RPC-B & \\
\hline Number of patients & 84 & 59 & 58 & \\
\hline Patient age, years (median) & $0-19(8)$ & $0-19(8)$ & $0-19(9.5)$ & 0.417 \\
\hline Gender (male:female) & $45: 39$ & $30: 29$ & $35: 23$ & 0.583 \\
\hline \multicolumn{5}{|l|}{ Diagnosis } \\
\hline Acute lymphoblastic leukemia & 38 & 32 & 27 & 0.539 \\
\hline Acute myeloid leukemia & 14 & 8 & 10 & 0.858 \\
\hline Myelodysplastic syndrome & 2 & 2 & 1 & 1.000 \\
\hline Aplastic anemia & 6 & 1 & 5 & 0.197 \\
\hline Hemophagocytic lymphohistiocytosis & 3 & 0 & 1 & 0.306 \\
\hline Non-Hodgkin's lymphoma & 6 & 3 & 5 & 0.687 \\
\hline Neuroblastoma & 3 & 2 & 3 & 0.816 \\
\hline Brain tumor & 2 & 2 & 1 & 1.000 \\
\hline Other & 10 & 9 & 5 & 0.534 \\
\hline
\end{tabular}

ABBREVIATIONS: PC, platelet concentrate; P-PC, regular PC in plasma; RPC-B, resuspended PC in BRS-A as plasma-replaced PC; RPC-M, resuspended $\mathrm{PC}$ in $\mathrm{M}$-sol as plasma-replaced $\mathrm{PC}$.

Kruska-Wallis test was used to evaluate the difference in patient ages between each group. Fisher's exact test was used to determine the difference in other characteristics of patients transfused with each PC product. 
Table 2. Frequency of ATRs and transfusion respons e of each PC transfusion

\begin{tabular}{|c|c|c|c|c|}
\hline Transfusion-related parameters & $\mathrm{P}-\mathrm{PC}$ & RPC-M & RPC-B & $\mathrm{p}$-value \\
\hline Number of patients who received each PC transfusion & 84 & 59 & 58 & \\
\hline Number of patients with ATR(s) & 12 & 1 & 4 & \\
\hline Ratio of ATRs per transfused patient & $14.3 \%$ & $1.7 \%$ & $6.9 \%$ & 0.022 \\
\hline Total number of transfused PC products & 679 & 1182 & 1044 & \\
\hline Number of products associated with ATR & 12 & 5 & 4 & \\
\hline Ratio of ATRs per transfused product & $1.8 \%$ & $0.4 \%$ & $0.4 \%$ & 0.003 \\
\hline \multicolumn{5}{|l|}{ Transfusion response } \\
\hline Evaluable number of transfusions & 113 & 147 & 140 & \\
\hline $\mathrm{CCI} 24 \mathrm{~h}\left(\times 10^{10} / \mathrm{L} ;\right.$ median $\left.\pm \mathrm{SD}\right)$ & $1.50 \pm 0.92$ & $1.38 \pm 0.72$ & $1.40 \pm 0.81$ & 0.269 \\
\hline
\end{tabular}

ABBREVIATIONS: ATR(s), allergic transfusion reaction(s); CCI 24h, post-transfusion corrected count increment after 24

hours; PC, platelet concentrate; P-PC, regular PC in plasma; RPC-B, resuspended PC in BRS-A as plasma-replaced PC; RPC-M, resuspended PC in M-sol as plasma-replaced PC.

ATRs were evaluated by the frequency of each incidence per patient and bag between P-PC and RPC with BRS-A or Msol. Fisher's exact test was used to assess the differences in the ATR ratio for the three different PC products. Kruskal-

Wallis test was used to evaluate the effect of the transfusion response in terms of 24-h CCI for each PC product. 
Table 3. Result of the quality inspection of each PC product

\begin{tabular}{|c|c|c|c|c|c|}
\hline \multicolumn{2}{|c|}{ Composition and platelet activation tests } & \multirow{2}{*}{$\begin{array}{c}\text { P-PC } \\
43\end{array}$} & \multirow{2}{*}{$\begin{array}{c}\text { RPC-M } \\
18\end{array}$} & \multirow{2}{*}{$\begin{array}{c}\text { RPC-B } \\
25\end{array}$} & \multirow[t]{2}{*}{ p-value } \\
\hline Number evaluated & & & & & \\
\hline Platelet recovery & $(\%)$ & - & $90.56 \pm 0.11$ & $88.49 \pm 0.05$ & 0.456 \\
\hline Plasma protein reduction & $(\%)$ & - & $95.78 \pm 0.01$ & $95.90 \pm 0.01$ & 0.884 \\
\hline $\mathrm{pH}$ & & $7.37 \pm 0.11$ & $7.46 \pm 0.18$ & $7.26 \pm 0.24$ & 0.004 \\
\hline Total protein & $(\mathrm{g} / \mathrm{dL})$ & $5.87 \pm 0.40$ & $0.24 \pm 0.05$ & $0.24 \pm 0.06$ & 0.864 \\
\hline Albmin & $(\mathrm{g} / \mathrm{dL})$ & $3.42 \pm 0.62$ & $0.19 \pm 0.03$ & $0.18 \pm 0.05$ & 0.361 \\
\hline $\mathrm{Na}$ & $(\mathrm{mmol} / \mathrm{L})$ & $159.40 \pm 1.81$ & $166.91 \pm 1.34$ & $136.40 \pm 1.60$ & $<0.001$ \\
\hline $\mathrm{K}$ & $(\mathrm{mmol} / \mathrm{L})$ & $3.14 \pm 0.24$ & $2.93 \pm 0.71$ & $3.88 \pm 0.08$ & $<0.001$ \\
\hline $\mathrm{Cl}$ & $(\mathrm{mmol} / \mathrm{L})$ & $80.61 \pm 2.19$ & $84.42 \pm 0.35$ & $103.18 \pm 1.18$ & $<0.001$ \\
\hline $\mathrm{Ca}$ & $(\mathrm{mg} / \mathrm{dL})$ & $7.53 \pm 0.26$ & $4.15 \pm 0.11$ & $5.69 \pm 0.11$ & $<0.001$ \\
\hline $\mathrm{P}$ & $(\mathrm{mg} / \mathrm{dL})$ & $2.76 \pm 0.46$ & $0.16 \pm 0.05$ & $0.23 \pm 0.07$ & 0.002 \\
\hline $\mathrm{Mg}$ & $(\mathrm{mg} / \mathrm{dL})$ & $1.78 \pm 0.13$ & $3.54 \pm 0.10$ & $2.24 \pm 0.05$ & $<0.001$ \\
\hline Glucose & $(\mathrm{mg} / \mathrm{dL})$ & $365.40 \pm 31.45$ & $265.72 \pm 6.06$ & $106.96 \pm 3.67$ & $<0.001$ \\
\hline
\end{tabular}

Abbreviations: PAS, platelet additive solution; PC, platelet concentrate; P-PC, regular PC in plasma; RPC-B, resuspended PC in BRS-A as plasma-replaced PC; RPC-M, resuspended PC in M-sol as plasma-replaced PC.

Unpaired t-test was used to analyze the difference in the quality inspection of RPC with two different PASs. P-value is for the comparison between RPC-M and RPC-B. 
Table4. Review of recent studies related to WPC, RPC, CPC, and PC in PAS transfusion in pediatric or neonatepopulations

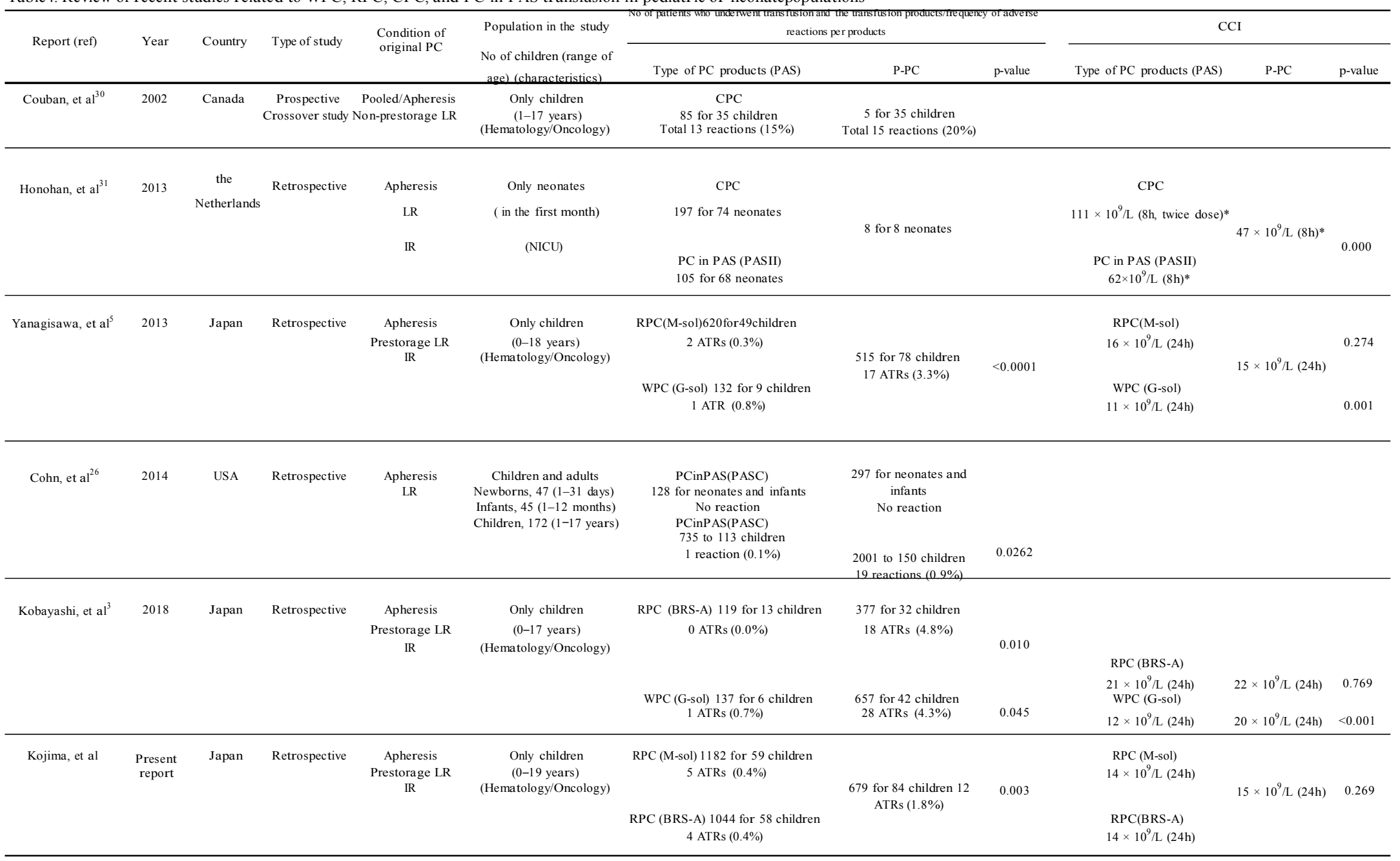

ABBREVIATIONS: ATR(s), allergic transfusion reaction(s); CCI, post-transfusion corrected count increment; CPC, concentrated or plasma reduced PC; IR, irradiated, LR, leukoreduced; NICU, neonatal intensive care unit; PAS, platelet additive solution; PC in PAS, plasma-replaced PC stored with PAS at residual plasma in $30 \%-35 \%$; PC(s), platelet concentrate(s); P-PC, regular PC in plasma; RPC, resuspended PC in PAS as plasma-replaced PC; WPC washed PC. *The scores were shown as count increments in the report. 


\section{References}

1. Azuma H, Hirayama J, Akino M, Miura R, Kiyama Y, Imai K, Kasai M, Koizumi K, Kakinoki Y, Makiguchi Y, Kubo K, Atsuta Y, Fujihara M, Homma $\mathrm{C}$, Yamamoto S, Kato T, Ikeda H. Reduction in adverse reactions to platelets by the removal of plasma supernatant and resuspension in a new additive solution (M-sol). Transfusion 2009;49: 214-8.

2. Azuma H, Ikeda H. Washed/Replaced Platelet Concentrates. Vox Sanguinis 2009;97: 38-.

3. Kobayashi J, Yanagisawa R, Ono T, Tatsuzawa Y, Tokutake Y, Kubota N, Hidaka E, Sakashita K, Kojima S, Shimodaira S, Nakamura T. Administration of platelet concentrates suspended in bicarbonated Ringer's solution in children who had platelet transfusion reactions. Vox Sang 2018;113: 128-35.

4. Tobian AA, Savage WJ, Tisch DJ, Thoman S, King KE, Ness PM. Prevention of allergic transfusion reactions to platelets and red blood cells through plasma reduction. Transfusion 2011;51: 1676-83.

5. Yanagisawa R, Shimodaira S, Kojima S, Nakasone N, Ishikawa S, Momose K, Honda T, Yoshikawa K, Saito S, Tanaka M, Nakazawa Y, Sakashita K, Shiohara M, Akino M, Hirayama J, Azuma H, Koike K. Replaced platelet concentrates containing a new additive solution, M-sol: safety and efficacy for pediatric patients. Transfusion 2013;53: 2053-60.

6. Gulliksson H. Platelet storage media. Vox Sang 2014;107: 205-12.

7. Hirayama J, Azuma H, Fujihara M, Akino M, Homma C, Kato T, Ikeda H. Comparison between in vitro qualities of platelets washed with commercially available additive solutions and those washed with M-sol. Vox Sanguinis 2010;99: 131-5.

8. Hirayama J, Azuma H, Fujihara M, Homma C, Yamamoto S, Ikeda H. Storage of platelets in a novel additive solution (M-sol), which is prepared by mixing solutions approved for clinical use that are not especially for platelet storage. Transfusion 2007;47: 960-5.

9. Oikawa S, Sasaki D, Kikuchi M, Sawamura Y, Itoh T. Comparative in vitro evaluation of apheresis platelets stored with $100 \%$ plasma versus bicarbonated Ringer's solution with less than 5\% plasma. Transfusion 2013;53: 655-60.

10. Oikawa S, Taguchi T, Endo K, Hoshi T, Kawashima W, Horibe Y, Urano S, Suzuki K, Minegishi M, Itoh T, Shimizu H. Storage of washed platelets in BRS-A platelet additive solutions based on two types of clinically available bicarbonated Ringer's solutions with different electrolyte concentrations. Transfus Apher Sci 2015;53: 233-7.

11. Iwama A, Hirayama J, Nogawa M, Shiba M, Satake M, Takamoto S, Tadokoro $\mathrm{K}$. Comparison between in vitro properties of washed platelet concentrates suspended in M-sol and those in BRS-A, both of which were prepared with an automated cell processor. Transfus Apher Sci 2017;56: 241-4.

12. Mishima Y, Tsuno NH, Matsuhashi M, Yoshizato T, Sato T, Ikeda T, Watanabe-Okochi N, Nagura Y, Sone S, Kurokawa M, Okazaki H. Effects of universal vs bedside leukoreductions on the alloimmunization to platelets and the platelet transfusion refractoriness. Transfus Apher Sci 2015;52: 112-21. 
13. Liumbruno GM, Catalano L, Piccinini V, Pupella S, Grazzini G. Reduction of the risk of bacterial contamination of blood components through diversion of the first part of the donation of blood and blood components. Blood Transfus 2009; 7: 86-93.

14. Nakamura A, Abe K, Masuya M, Imai S, Ohishi K, Mori Y, Kojima T, Wada H, Katayama N, Nobori T. Efficiency of diversion of the first aliquot of blood and prestorage leukoreduction for preventing bacterial contamination in red blood cell concentrates assessed using a rapid polymerase chain reaction-based bacterial detection system. Transfus Med 2011;21: 365-70.

15. Satake M, Mitani T, Oikawa S, Nagumo H, Sugiura S, Tateyama H, Awakihara S, Mitsutomi Y, Muraoka M, Tadokoro K. Frequency of bacterial contamination of platelet concentrates before and after introduction of diversion method in Japan. Transfusion 2009;49: 2152-7.

16. Yanagisawa R, Shimodaira S, Sakashita K, Hidaka Y, Kojima S, Nishijima F, Hidaka E, Shiohara M, Nakamura T. Factors related to allergic transfusion reactions and febrile non-haemolytic transfusion reactions in children. Vox Sang 2016;110: 376-84.

17. Kanda Y. Investigation of the freely available easy-to-use software 'EZR' for medical statistics. Bone Marrow Transplant 2013;48: 452-8.

18. Karafin M, Fuller AK, Savage WJ, King KE, Ness PM, Tobian AA. The impact of apheresis platelet manipulation on corrected count increment. Transfusion 2012;52: 1221-7.

19. de Wildt-Eggen J, Schrijver JG, Bins M, Gulliksson H. Storage of platelets in additive solutions: effects of magnesium and/or potassium. Transfusion 2002;42: 76-80.

20. van der Meer PF. PAS or plasma for storage of platelets? A concise review. Transfus Med 2016;26: 339-42.

21. Wagner SJ, Skripchenko A, Myrup A, Thompson-Montgomery D, Awatefe H, Moroff G. Calcium is a key constituent for maintaining the in vitro properties of platelets suspended in the bicarbonate-containing additive solution M-sol with low plasma levels. Transfusion 2010;50: 1028-35.

22. Fontaine MJ, Shih H, Schubert R, Wong W, Andrews J, Jeng M, Tirouvanziam R. Leukocyte and plasma activation profiles in chronically transfused patients with a history of allergic reactions. Transfusion 2017;57: 2639-48.

23. Oakley FD, Woods M, Arnold S, Young PP. Transfusion reactions in pediatric compared with adult patients: a look at rate, reaction type, and associated products. Transfusion 2015;55: 563-70.

24. Vossoughi S, Perez G, Whitaker BI, Fung MK, Stotler B. Analysis of pediatric adverse reactions to transfusions. Transfusion 2018;58: 60-9.

25. Buck SA, Kickler TS, McGuire M, Braine HG, Ness PM. The utility of platelet washing using an automated procedure for severe platelet allergic reactions. Transfusion 1987;27: 391-3.

26. Cohn CS, Stubbs J, Schwartz J, Francis R, Goss C, Cushing M, Shaz B, Mair D, Brantigan B, Heaton WA. A comparison of adverse reaction rates for PAS C versus plasma platelet units. Transfusion 2014;54: 1927-34.

27. de Wildt-Eggen J, Nauta S, Schrijver JG, van Marwijk Kooy M, Bins M, van Prooijen HC. Reactions and platelet increments after transfusion of platelet concentrates in plasma or an additive solution: a prospective, randomized study. Transfusion 2000;40: 398-403. 
28. Heddle NM, Blajchman MA, Meyer RM, Lipton JH, Walker IR, Sher GD, Constantini LA, Patterson B, Roberts RS, Thorpe KE, Levine MN. A randomized controlled trial comparing the frequency of acute reactions to plasma-removed platelets and prestorage WBC-reduced platelets. Transfusion 2002;42: 556-66.

29. Tobian AA, Fuller AK, Uglik K, Tisch DJ, Borge PD, Benjamin RJ, Ness PM, King KE. The impact of platelet additive solution apheresis platelets on allergic transfusion reactions and corrected count increment (CME). Transfusion 2014;54: 1523-9; quiz 2.

30. Couban S, Carruthers J, Andreou P, Klama LN, Barr R, Kelton JG, Heddle NM. Platelet transfusions in children: results of a randomized, prospective, crossover trial of plasma removal and a prospective audit of WBC reduction. Transfusion 2002;42: 753-8.

31. Honohan A, van't Ende E, Hulzebos C, Lopriore E, van't Verlaat E, Govaert P, Brand A, van der Bom J. Posttransfusion platelet increments after different platelet products in neonates: a retrospective cohort study. Transfusion 2013;53: 3100-9.

32. Vo TD, Cowles J, Heal JM, Blumberg N. Platelet washing to prevent recurrent febrile reactions to leucocyte-reduced transfusions. Transfus Med 2001;11: 45-7.

33. Sahai T, Henrichs K, Refaai M, Heal JM, Kirkley SA, Schmidt AE, Mendler JH, Masel D, Liesveld J, Aquina C, Blumberg N. ABO identical and washed blood transfusions as candidate strategies to reduce early mortality in acute promyelocytic leukemia. Leuk Res 2017;62: 1-3.

34. Oikawa S, Minegishi M, Endo K, Kawashima W, Suzuki K, Shimizu H. Washing of platelets can be fully automated using a closed-system cell processor and BRS-A platelet additive solution. Vox Sang 2016;111: 437-40. 